Pesq. Vet. Bras. 20(3):102-108, jul./set. 2000

\title{
Intoxicação experimental por monensina em eqüinos ${ }^{1}$
}

\author{
Pedro Soares Bezerra $\mathrm{Jr}^{2}$, Marcia Regina Silva Ilha ${ }^{3}$, Ingeborg Maria Langohr ${ }^{3}$ e \\ Claudio S.L. Barros ${ }^{4}$
}

\begin{abstract}
Bezerra Jr P.S., Ilha M.R.S, Langohr I.M. \& Barros C.S.L. 2000. [Experimental monensin poisoning in horses.] Intoxicação experimental por monensina em eqüinos. Pesquisa Veterinária Brasileira 20(3):102-108. Depto Patologia, Universidade Federal de Santa Maria, 97105900 Santa Maria, RS, Brazil. E-mail: cslb@sm.conex.com.br

Seven horses were experimentally treated with sodium monensin. Two of them were fed 3-4 kg/horse/day with a commercial ration (containing $180 \mathrm{ppm} \pm 20$ ) known to be implicated in natural outbreaks of monensin poisoning in horses. A third horse was fed a single dose of $5 \mathrm{mg} / \mathrm{kg}$ and another one received four daily doses of $1 \mathrm{mg} / \mathrm{kg}$ of sodium monensin (from a premix source). These four horses died or were euthanitised in extremis 3-8 days after the beginning of administration of the drug. A fifth horse was fed a single dose of $5 \mathrm{mg} / \mathrm{kg}$ of monensin, became mildly ill but recovered. Two experimental horses did not develop signs of the toxicosis. One of them was fed 40 daily doses of $0.5 \mathrm{mg} / \mathrm{kg}$ of monensin. The other was fed $3 \mathrm{~kg} /$ day of the same brand of ration used in farms where outbreaks of monensin poisoning in horses have been reported (but from another batch later determined to have monensin levels of only $<5 \mathrm{ppm}$ ). The onset of clinical signs varied from 2 to 5 days after administration of the drug, and clinical courses lasted from 24 to 76 hours. Clinical signs included tachycardia and cardiac arythmia, groaning, incoordination, sudoresis, sternal or lateral recumbency, paddling and death. Marked increases in the plasmatic activity of creatine phosphokinase were observed in five poisoned horses, and a mild increase in the aspartate aminotransferase plasmatic activity was detected in one of the animals. Main necropsy findings were in the skeletal muscles and consisted of focal to focally extensive white or yellow areas of discoloration with bilateral symmetric distribution. These lesions were associated with gelatinous transluscent edema in the intermuscular fasciae. More intensively affected muscles included quadriceps femoris, adductor, pectineus, gracilis, semimembranous, supraspinatus, subscapularis and brachycephalic. Histopathological findings consisted of segmentar, multifocal to coalescent, degenerative myopathy. Both gross and microscopic lesions were absent in the skeletal muscles from two of the horses fed the monensin premix [5 mg/ $\mathrm{kg}$ (single dose) and $4 \mathrm{mg} / \mathrm{kg}$ (four daily doses of $1 \mathrm{mg} / \mathrm{kg}$ )]. Neither gross nor microscopic lesions were observed in the myocardium of any of these horses.
\end{abstract}

INDEX TERMS: Ionophore antibiotics, monensin, disease of horses, pathology, myopathy.

RESUMO.- Sete eqüinos foram tratados experimentalmente com monensina sódica. Dois desses animais receberem 3-4 $\mathrm{kg} / \mathrm{equïno} /$ dia de uma ração comercial sabidamente implicada em surtos naturais da intoxicação por monensina em eqüinos e que continha $180 \mathrm{ppm} \pm 20$ da droga. Um equïno recebeu

\footnotetext{
${ }^{1}$ Aceito para publicação em 9 de março de 2000.

Parte da tese de mestrado do primeiro autor, defendida na Universidade Federal de Santa Maria (UFSM), em 12 de março de 1999.

2Programa de Pós-Graduação em Medicina Veterinária, Área de Concentração em Patologia Veterinária, Centro de Ciências Rurais, UFSM.
}

uma única dose de $5 \mathrm{mg} / \mathrm{kg}$ e um outro recebeu 4 doses diárias de $1 \mathrm{mg} / \mathrm{kg}$ de monensina sódica originária de um premix. Esses quatro eqüinos morreram ou foram sacrificados in extremis, 3-8 dias após o início da administração da droga. Um quinto eqüino recebeu dose única de $5 \mathrm{mg} / \mathrm{kg}$ de

\footnotetext{
${ }^{3}$ Estagiária do Departamento de Patologia da UFSM; bolsista de Iniciação Científica do $\mathrm{CNPq} / \mathrm{PIBIC}$.

${ }^{4}$ Departamento de Patologia, UFSM, 97105-900 Santa Maria, Rio Grande do Sul. E-mail: cslb@sm.conex.com.br
} 
monensina, ficou levemente doente e se recuperou. Dois eqüinos não desenvolveram sinais da intoxicação. Um desses eqüinos tinha recebido 40 doses diárias de $0,5 \mathrm{mg} / \mathrm{kg}$ de monensina e o outro recebeu $3 \mathrm{~kg} / \mathrm{dia}$ de uma ração da mesma marca que a usada nas fazendas onde surtos de intoxicação por monensina foram detectados (mas de uma outra partida, mais tarde determinada como contendo menos de 5 ppm de monensina). $O$ aparecimento dos sinais clínicos ocorreu de 2 a 5 dias após a administração da droga e a duração do quadro clínico variou de 24 a 76 horas. Os sinais clínicos incluíam taquicardia, arritmia, gemidos, incoordenação, sudorese, decúbito esternal, decúbito lateral, pedaleios e morte. Em cinco dos eqüinos intoxicados observaram-se marcadas elevações da atividade plasmática de creatina fosfoquinase e, em um equiino, houve leve aumento da atividade plasmática de aspartato aminotransferase. Os principais achados de necropsia consistiram em áreas brancas ou amarelas, focais ou focalmente extensas e bilateralmente simétricas nos músculos esqueléticos. Essas lesões eram associadas a edema gelatinoso e translúcido das fáscias intermusculares. Quadríceps femoral, adutor, pectíneo, grácil, semimembranáceo, supra-espinhal, subescapular, braquicefálico e quadríceps femoral estavam entre os músculos esqueléticos mais afetados. Os exames histológicos revelaram miopatia segmentar, multifocal a coalescente. Em dois eqüinos que receberam o premix de monensina [5 $\mathrm{mg} / \mathrm{kg}$ (dose única) e 4 $\mathrm{mg} / \mathrm{kg}$ (quatro doses diárias de $1 \mathrm{mg} / \mathrm{kg}$ )] não se observaram lesões macroscópicas ou microscópicas. Em nenhum dos dois eqüinos se observaram lesões macro ou microscópicas no miocárdio.

TERMOS DE INDEXAÇÃO: Antibióticos ionóforos, monensina, doenças de eqüinos, patologia, miopatia.

\section{INTRODUÇÃO}

Antibióticos ionóforos (AIs) formam complexos bipolares irreversíveis lipossolúveis com cátions $\left(\mathrm{Ca}^{++}, \mathrm{K}^{+}, \mathrm{Mg}^{++}\right)$, favorecendo o transporte iônico através de membranas biológicas com resultante perturbação da homeostase iônica intracelular. Os Als são usados como coccidiostáticos para várias espécies animais, e para promoção de crescimento e prevenção de timpanismo em bovinos (Barros 1998). Embora considerados seguros quando usados para a espécie-alvo, dentro das doses recomendadas pelo fabricante, o uso dessas drogas tem sido responsável por intoxicação em bovinos (Gava et al. 1997), eqüinos (Bezerra et al. 1999), ovinos (RietCorrea et al. 1999), suínos (Armién et al. 1997), coelhos (Salles et al.1994), cães (Wilson 1980), galinhas (Chalmers 1981) e outras aves (Gregory et al. 1992). Miopatia e miocardiopatia degenerativas são as principais lesões observadas nos animais afetados (Barros 1998).

Monensina, uma substância produzida pelo fungo Streptomyces cinnamonensis, é um dos Als mais usados em medicina veterinária. Eqüinos são particularmente sensíveis a essa droga $\left(\mathrm{DL}_{50}=2-3 \mathrm{mg} / \mathrm{kg}\right)$ e recentemente três surtos da intoxicação foram descritos nessa espécie no Rio Grande do Sul (Bezerra et al. 1999). Por essa razão, um estudo da intoxicação experimental por monensina foi realizado em eqüinos com o objetivo de determinar o quadro clínico, achados de necropsia e histopatologia.

\section{MATERIAL E MÉTODOS}

Os dados sobre os sete eqüinos do experimento encontram-se no Quadro 1. Dois eqüinos (eqüinos $\mathrm{A}$ e $\mathrm{C}$ ) receberam respectivamente 4 e $3 \mathrm{~kg} /$ dia da ração responsável por um surto de intoxicação espontânea por monensina em eqüinos (Bezerra et al. 1999). O conteúdo dessa ração foi determinado como $180 \pm 20 \mathrm{ppm}^{5}$. Um outro eqüino (eqüino $B$ ) recebeu $3 \mathrm{~kg} /$ dia de uma outra partida da ração de mesma marca, adquirida no comércio $(\mathrm{RC})$, após a ocorrência do surto e cujo conteúdo de monensina foi posteriormente determinado como abaixo de 5 ppm. Essas administrações visaram estabelecer o diagnóstico do surto espontâneo da intoxicação (Bezerra et al. 1999). Um premix contendo $20 \%$ de monensina sódica ${ }^{6}$ foi administrado a outros quatro eqüinos por sonda através de sonda nasogástrica diluído em água (equiino D) ou no cocho misturado ao alimento (eqüinos $\mathrm{E}, \mathrm{F}$ e $\mathrm{G}$ ). As administrações de doses baixas múltiplas realizadas nos eqüinos $\mathrm{F}$ e $\mathrm{G}$ visavam verificar um possível poder cumulativo da droga. As quantidades de monensina consumidas pelos eqüinos $\mathrm{A}$ e $\mathrm{C}$ foram calculadas medindo $\mathrm{o}$ consumo diário da ração. As quantidades de monensina administrada a cada eqüino constam no Quadro 1.

Amostras de sangue com anticoagulante (EDTA) foram colhidas diariamente para determinação da atividade plasmática das enzimas creatina fosfoquinase (CK) e aspartato aminotransferase (AST). A atividade plasmática da $\mathrm{CK}$ foi avaliada pelo método de espectrofotometria contínua IFCC $^{7}$ e a da AST, pelo método colorimétrico de Reitmam e Frankel ${ }^{8}$.

Dos sete eqüinos do experimento dois tiveram morte espontânea e dois foram sacrificados in extremis com barbitúricos. Todos foram necropsiados e fragmentos de diversos órgãos foram fixados em formol a $10 \%$ e processados rotineiramente para histopatologia e corados pela hematoxilina e eosina (HE). Consistentemente foram colhidos fragmentos de 27 músculos diferentes e fragmentos de miocárdio do bordo livre das quatro câmaras cardíacas e do septo interventricular.

\section{RESULTADOS}

\section{Sinais clínicos}

Dos sete eqüinos do experimento, dois tiveram morte espontânea, dois foram sacrificados quando moribundos, um adoeceu levemente e se recuperou e dois não apresentaram sinais clínicos. $O$ início dos sinais clínicos variou de 2 a 5 dias e a duração do quadro clínico, de 24 a 76 horas. Os dados relativos às doses, número de administrações, início e duração dos sinais clínicos e desfecho da intoxicação para cada eqüino constam no Quadro 1.

\footnotetext{
${ }^{5}$ Determinações realizadas pelo Prof. Dr. J. Kamphues, Institut für Tierernährung der Tierärztlichen Hochschule Hannover, Bischofsholer Damm 15, D-30173 Hannover, Alemanha.

${ }^{6}$ Coban 200 (monensina a 20\%), ELANCO, Av. Morumbi 8264, 04703-002 São Paulo, SP.

${ }^{7}$ Biosystems S.A. Costa Brava 30-4, 08030 Barcelona, Espanha.

${ }^{8}$ QUIBASA-Química Básica Ltda, Rua Teles de Menezes 92, 31565-130 Belo Horizonte, MG.
} 
Quadro 1. Intoxicação experimental por monensina em eqüinos

\begin{tabular}{|c|c|c|c|c|c|c|c|c|}
\hline Eqüino & Sexo & Idade $^{\mathrm{a}}$ & $\begin{array}{l}\text { Dose } \\
\text { diária } \\
(\mathrm{mg} / \mathrm{kg})\end{array}$ & $\begin{array}{l}\text { Número de } \\
\text { administra- } \\
\text { ções diárias }\end{array}$ & $\begin{array}{c}\text { Dose } \\
\text { total } \\
(\mathrm{mg} / \mathrm{kg})\end{array}$ & $\begin{array}{l}\text { Início dos } \\
\text { sinais } \\
\text { clínicos } \\
\text { após adm. } \\
\text { (dias) }\end{array}$ & $\begin{array}{c}\text { Duração } \\
\text { dos } \\
\text { sinais } \\
\text { clínicos } \\
\text { (horas) }\end{array}$ & Desfecho \\
\hline A & $M C^{b}$ & $\begin{array}{c}15 \\
\text { anos }\end{array}$ & $1,78^{\mathrm{c}}$ & 10 & 17,8 & 5 & 76 & Morreu \\
\hline B & $\mathrm{F}$ & $\begin{array}{c}6 \\
\text { meses }\end{array}$ & $-d$ & - & - & 2 & 56 & Morreu \\
\hline C & $\mathrm{F}$ & $\begin{array}{c}7 \\
\text { meses }\end{array}$ & 2,48 & 6 & 14,85 & 5 & 48 & $\begin{array}{c}\text { Adoeceu } \\
\text { levemente e } \\
\text { recuperou-se }\end{array}$ \\
\hline D & $\mathrm{F}$ & $\begin{array}{c}7 \\
\text { anos }\end{array}$ & 5 & 1 & 5 & 2 & 24 & Sacrificado \\
\hline E & $\mathrm{F}$ & $\begin{array}{c}15 \\
\text { anos }\end{array}$ & 5 & 1 & 5 & 3 & 32 & Sacrificado \\
\hline $\mathrm{F}$ & MC & $\begin{array}{c}8 \\
\text { anos }\end{array}$ & 1 & 4 & 4 & - & - & Não adoeceu \\
\hline G & MC & $\begin{array}{c}8 \\
\text { anos }\end{array}$ & 0,5 & 40 & 20 & & & \\
\hline
\end{tabular}

Quadro 2. Principais sinais clínicos na intoxicação experimental por monensina em eqüinos

\begin{tabular}{lcccccc}
\hline \multirow{2}{*}{ Sinal clínico } & \multicolumn{7}{c}{ Eqüino } \\
\cline { 2 - 6 } & A & C & D & E & F \\
\hline Anorexia parcial & $++^{\mathrm{a}}$ & + & + & + & + \\
Apatia & + & + & + & + & + \\
Arrastamento de pinças & + & + & + & - & + \\
Tropeços & - & + & - & + & + \\
Incoordenação dos membros pélvicos & + & + & - & - & + \\
Andar rígido & - & + & - & - & - \\
Relutância em andar & + & - & - & - & - \\
Troca freqüente de apoio & - & + & - & - & - \\
Deitar e levantar repetidos e com dificuldade & - & + & - & - & - \\
Decúbito permanente & + & + & - & + & + \\
Alternância de decúbitos external e lateral & - & + & - & + & - \\
Congestão das mucosas & + & + & - & - & + \\
Sudorese & + & + & - & - & - \\
Taquicardia & + & + & - & - & - \\
Respiração ofegante & - & + & - & - & - \\
Sons abdominais diminúdos & - & + & - & - & + \\
& & & & & &
\end{tabular}

a+ Presença, - ausência de sinal clínico.

Todos os animais que adoeceram apresentaram inicialmente redução temporária do apetite. Os eqüinos $\mathrm{A}, \mathrm{C}$ e E consumiam lentamente o alimento com monensina, entretanto comiam bem o feno de alfafa. Os equiinos $\mathrm{D}$ e $\mathrm{F}$ diminuíram também o consumo de feno de alfafa temporariamente.

Os principais sinais clínicos apresentados pelos eqüinos estão agrupados no Quadro 2. Todos os eqüinos que adoeceram mostraram inicialmente leve apatia. Quando forçados a trotar tinham incoordenação dos membros pélvicos (equiinos A, C e F), observada inicialmente apenas quando mudavam repentinamente de direção (equiino F), arrastavam as pinças dos membros pélvicos (eqüinos $\mathrm{A}, \mathrm{C}$, D e F) e tropeçavam (equiinos $\mathrm{C}$, $\mathrm{E}$ e $\mathrm{F}$ ). $\mathrm{Em}$ seguida apresentavam andar rígido (equiino $\mathrm{C}$ ), relutância em andar (eqüino $\mathrm{A}$ ), troca constante de apoio (eqüino $\mathrm{C}$ ), deitar e levantar repetidos e com dificuldade (equiino $\mathrm{C}$ ), até se tornarem incapazes de levantar, permanecendo em decúbito (eqüinos A, C, E e F) (Fig. 1). Constantemente alternavam decúbitos esternal e lateral (equiinos

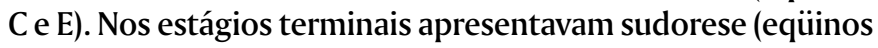
A e C), congestão das mucosas (eqüinos A, C e F), taquicardia (equiinos A e C), respiração ofegante (eqüino C), sons abdominais diminuídos ou ausentes (equiinos $\mathrm{Ce} F$ ), gemidos (equiinos $\mathrm{C}$ e $\mathrm{F}$ ), ranger de dentes (equiinos $\mathrm{C}$ e $\mathrm{F}$ ) e pedaleios (equiinos $\mathrm{C}$ e F). Os eqüinos $\mathrm{C}$ e $\mathrm{F}$ apresentavam hipotermia próximo a morte.

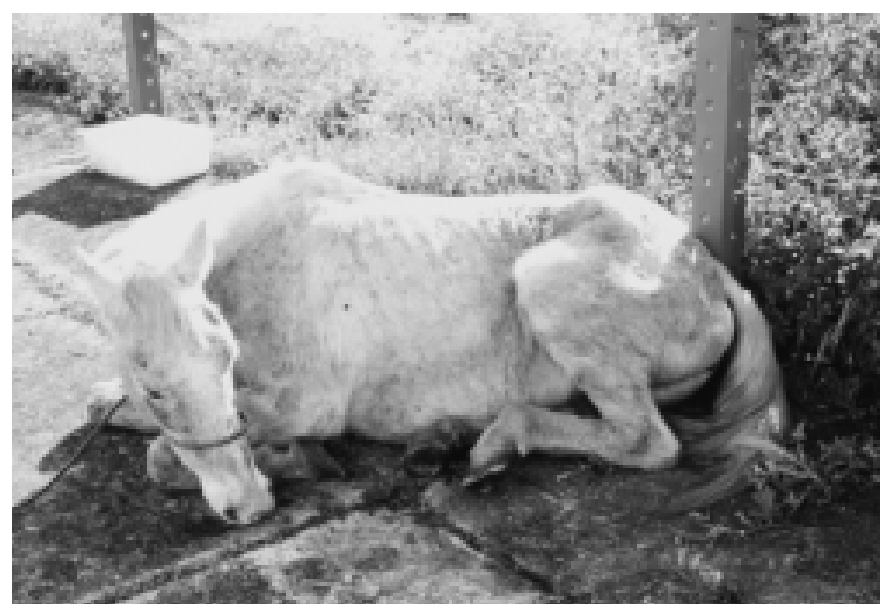

Fig. 1. Decúbito permanente algumas horas antes da morte, na intoxicação experimental por monensina (Eqüino F). 
Houve elevação da atividade da CK nos eqüinos A, C, D, F e $\mathrm{G}$ com valores máximos de $2.341 \mathrm{UI} / \mathrm{L}$ a $142.240 \mathrm{UI} / \mathrm{L}$. $\mathrm{O}$ equiino D apresentou leve elevação na atividade sérica de AST. Esta enzima esteve inalterada nos outros eqüinos.

\section{Achados de necropsia}

Os principais achados de necropsia foram observados nos músculos esqueléticos e consistiam de áreas ou estrias branco amareladas focalmente extensas ou difusas, bilaterais e simétricas. As lesões variaram de acentuadas (eqüino $\mathrm{C}$ ) a leves (eqüino $\mathrm{A}$ ). Dois eqüinos (E e $\mathrm{F}$ ) não apresentavam alterações macroscópicas nos músculos esqueléticos. As lesões acentuadas eram geralmente associadas a edema gelatinoso e translúcido entre as fáscias musculares. Os principais músculos com lesões macroscópicas eram o quadríceps femoral, adutor, pectíneo, grácil, semimembranáceo, supra-espinhal, subescapular, braquicefálico e quadríceps femoral.

Havia petéquias e sufusões no epicárdio e hidropericárdio dos equiinos $\mathrm{A}$ e $\mathrm{C}$.

Histopatologia dos músculos esqueléticos e do miocárdio

As lesões microscópicas nos músculos esqueléticos caracterizavam-se por miopatia necrosante segmentar, multifocal ou coalescente, associada a processos regenerativos das miofibras. Nos cortes longitudinais dos músculos, eram observados segmentos de miofibras com tumefação, aumento da eosinofilia, perda das estriações transversais e longitudinais (necrose hialina). Esses segmentos eram contíguos a segmentos normais e, em cortes transversais, eram arredondados ao invés de apresentarem a forma poligonal normal. A fragmentação do sarcoplasma desses segmentos formava aglomerados de detritos eosinofílicos irregulares (necrose flocular) (Fig. 2). Quando essas lesões eram acentuadas, freqüientemente estavam associadas a edema intersticial moderado ou acentuado. As lesões regenerativas eram iniciadas por infiltrado inflamatório constituído por proporções semelhantes de neutrófilos e macrófagos em áreas de necrose hialina e flocular. Os macrófagos em geral apresentavam citoplasma abundante e eosinofílico, indicando fagocitose de

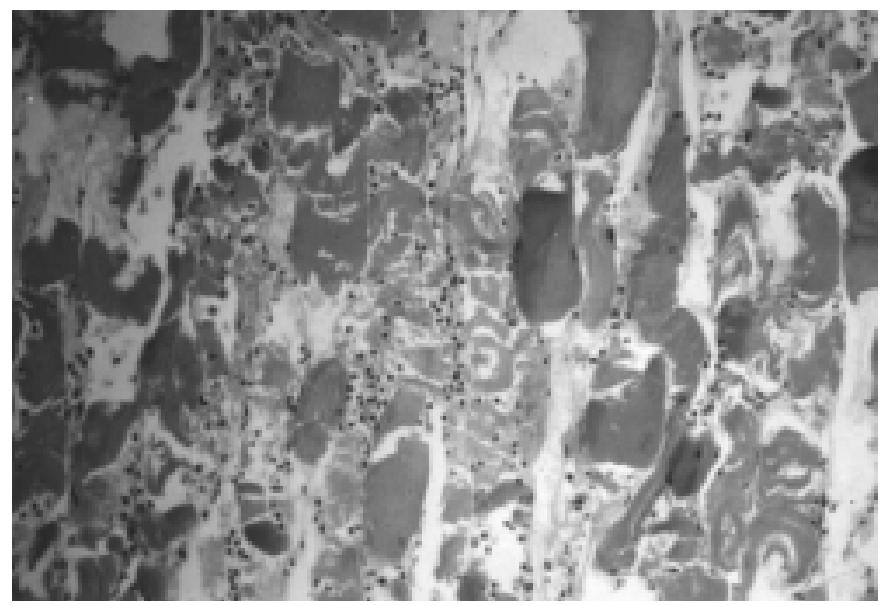

Fig. 2. Fragmentação do sarcoplasma de miofibras, na intoxicação experimental por monensina (Eqüino C). HE, obj. 40. restos sarcoplasmáticos. Raramente células gigantes multinucleadas eram observadas no interior de tubos sarcolemais. Ocasionalmente, as células satélites adjacentes a segmentos necróticos estavam tumefeitas com núcleos vesiculares e nucléolos proeminentes. Nas áreas de necrose flocular, essas células ocorriam no interior do tubo sarcolemal e eram irregulares ou alongadas, possuíam núcleos vesiculares, nucléolos proeminentes e citoplasma abundante levemente basofílico. Alterações histológicas estavam ausentes nos músculos esqueléticos dos equiinos $\mathrm{E}$ e $\mathrm{F}$ e do miocárdio de todos os equiinos.

\section{DISCUSSÃO}

A intoxicação produziu nos eqüinos deste experimento uma doença caracterizada principalmente por sinais clínicos e lesões anatomopatológicas referentes aos músculos esqueléticos.

Os sinais clínicos de distúrbios locomotores foram predominantes. Sinais clínicos semelhantes são descritos na maioria dos casos de intoxicação por Als e incluem incapacitação muscular (associados ou não a sinais de insuficiência cardíaca), algumas vezes precedidos por sinais de cólica. Sinais clínicos de distúrbios locomotores estiveram presentes também em dois eqüinos (E e F) embora não fossem observadas lesões macro ou microscópicas nos músculos esqueléticos nem no sistema nervoso central desses dois animais. Casos semelhantes têm sido descritos (Matsuoka 1976, Ordidge et al. 1979, McCracken et al. 1998) e os sinais clínicos atribuídos a distúrbios neurológicos (Matsuoka 1976). Porém esses autores não realizaram exame histológico do sistema nervoso. Os sinais clínicos relacionados à musculatura esquelética (mesmo na ausência de lesões macro e microscópicas) podem ser resultantes do efeito dos Als na distribuição dos íons sódio, potássio e cálcio nas miofibras. Distúrbios iônicos podem afetar a função muscular, pois os íons cálcio são essenciais para processos como transmissão neuromuscular e ativação e regulação da contração muscular (Amend et al. 1980).

Entre os outros sinais clínicos observaram-se sudorese, taquicardia, e respiração ofegante. A sudorese observada é um sinal freqüientemente relatado na intoxicação por AIs em eqüinos (Whitlock et al. 1978, Hanson et al. 1981, Rollinson et al. 1987). O mecanismo da sudorese nos equiinos é diferente do das demais espécies, as glândulas sudoríparas são inervadas por nervos simpáticos em que adrenalina é o neurotransmissor. Excitação e dor podem causar sudorese sem que ocorra aumento da temperatura corporal (Radostits et al. 1994). Desse modo, a sudorese pode não ser causada diretamente pela ação dos Als.

Distúrbios respiratórios são também relatados com frequiência na intoxicação por AIs em eqüinos (Gerhards et al. 1986, Doonan et al. 1989, Whitlock 1990). Tais sinais são atribuídos a miopatia degenerativa nos músculos intercostais e diafragma ou a edema pulmonar (Van Amstel \& Guthrie 1986). $\mathrm{O}$ eqüino $\mathrm{C}$, que apresentou respiração ofegante, tinha lesões acentuadas nos músculos intercostais e leves no diafragma que justificariam este sinal, já que não foram vistas alterações nos pulmões de nenhum eqüino deste estudo. Entre- 
tanto, o equiino A apresentou alterações semelhantes nesses músculos e não teve distúrbios respiratórios notáveis, pelo menos até 6 horas antes da morte quando foi examinado clinicamente pela última vez.

As atividades plasmáticas das enzimas creatina fosfoquinase (CK) e aspartato aminotransferase (AST) são caracteristicamente aumentadas após miopatias (Radostits et al. 1994, Valberg 1996). A atividade da CK esteve elevada em 4 dos 5 equiinos do experimento que adoeceram (eqüinos $\mathrm{A}, \mathrm{C}$, D e F), atingindo valores máximos de $2.300 \mathrm{UI} / \mathrm{L}$ a até $142.000 \mathrm{UI} /$ L. A AST esteve elevada apenas no eqüino D, e assim mesmo de modo leve. Nesse eqüino, a atividade da CK se elevou 5 dias após a administração da monensina, retornando aos valores normais em 6 dias. A atividade da AST excedeu os limites normais 10 dias após a administração, retornando à normalidade em 5 dias. A CK é uma enzima citoplasmática, por isso é liberada rapidamente em lesões iniciais. Já a AST, sendo ligada a membrana, é liberada mais tardiamente, somente quando a lesão se torna mais grave (Valberg 1996). Aumentos nas atividades destas enzimas são relatados com frequiência na intoxicação por AIs em eqüinos (Whitlock et al. 1978, Boemo et al. 1991, Kamphues et al. 1990, Hausmann \& Sasse 1991, Nicpon et al. 1997). Embora não fossem determinadas as isoenzimas da CK, é muito provável que a envolvida nos eqüinos do experimento seja a muscular, uma vez que não foram vistas alterações no miocárdio desses animais. Desse modo, a determinação da atividade sérica de CK deve ser o método diagnóstico auxiliar de escolha nos casos agudos da intoxicação por Als em eqüinos.

É interessante notar que dois eqüinos não apresentaram lesões macro ou microscópicas nos músculos esqueléticos e que nenhum dos equïnos do experimento apresentou lesões no miocárdio, ao contrário do que é descrito normalmente em eqüinos intoxicados por Als em que as lesões podem ser restritas ao miocárdio (Muylle et al. 1981), predominantes no miocárdio (Van de Kerk 1978), predominantes nos músculos esqueléticos (Van Amstel \& Guthrie 1986) ou afetar ambos igualmente (Gerhards et al. 1986). No entanto lesões restritas aos músculos esqueléticos, como observado em eqüinos deste estudo, não têm sido relatadas. $O$ fato de ocorrerem casos de intoxicação sem lesões musculares esqueléticas e/ ou cardíacas, e de não ocorrerem lesões em todos os músculos esqueléticos em todos os casos é um dado importante a ser considerado no diagnóstico da intoxicação a campo. É, portanto, indicada a realização do maior número de necropsias possível e a coleta de diversos músculos esqueléticos de cada animal em casos suspeitos de intoxicação por AIs. A distribuição das lesões encontradas nos eqüinos deste estudo podem servir de orientação para a escolha das amostras a serem examinadas microscopicamente.

A causa da morte nos eqüinos intoxicados por Als é geralmente atribuída à insuficiência cardíaca associada à cardiomiopatia degenerativa (Novilla 1992). Entretanto, sinais de insuficiência cardíaca podem ser encontrados mesmo em animais sem evidências macro ou microscópicas de lesões no miocárdio. Tais casos são atribuídos a lesões ultraestruturais afetando principalmente as mitocôndrias do miocárdio (Mollenhauer et al. 1981, 1984), a distúrbios bioquímicos associados a hipercalemia, à liberação de potássio dos músculos esqueléticos lesados (Nuytten et al. 1981) ou ao excesso de cálcio no sarcoplasma das miofibras provocado pelos AIs (Amend et al. 1980). Insuficiência respiratória causada por miopatia degenerativa nos músculos intercostais e diafragmáticos (Van Amstel \& Guthrie 1986) pode também contribuir para a morte.

O diagnóstico diferencial da intoxicação por Als em equiinos deve incluir principalmente a rabdomiólise de esforço, deficiência de vitamina E e selênio e intoxicação por Senna occidentalis (fedegoso). Dada a semelhança das lesões musculares, a epidemiologia e a análise química da ração são fatores importantes na conclusão do diagnóstico (Novilla 1992).

Na diferenciação da rabdomiólise de esforço, a avaliação do histórico é fundamental. Tipicamente, essa condição ocorre após trabalho ou treinamento que se seguem a um período de repouso com alimentação completa. Mioglobinúria é um achado comum. As lesões geralmente se concentram nos músculos da região glútea e lombar, podendo ser disseminadas. Os músculos são úmidos, tumefeitos e escuros. No miocárdio algumas vezes há alterações degenerativas leves (Hulland 1993). Na intoxicação por AIs, os sinais locomotores surgem sem associação ao exercício e as lesões musculares são observadas principalmente nos membros pélvicos (Nuytten et al. 1981), mas podem ter uma distribuição mais ampla, afetando os membros pélvicos e torácicos. A miopatia por armazenamento de polissacarídeos tem sido apontada como uma das causas de rabdomiólise por exercícios em várias raças de eqüinos. Entretanto, na histopatologia esta enfermidade é caracterizada por acúmulo de material PAS positivo dentro de miofibras e histiócitos (Beech 1997).

A deficiência de vitamina $E$ e de selênio ocorre principalmente em potros, podendo por vezes afetar animais adultos mal nutridos. As lesões podem ser extensas, afetando vários músculos, mas, freqüientemente, afetam ou se restringem aos músculos mastigatórios. Esteatite comumente acompanha as lesões de miopatia em potros, embora, ao que parece, não ocorra em adultos (Rooney \& Robertson 1996). Na deficiência de vitamina $\mathrm{E}$ e de selênio as miofibras podem apresentar graus elevados de mineralização (Hulland 1993). Por outro lado, a intoxicação por Als afeta eqüiinos de várias faixas etárias, independente do estado nutricional. As lesões podem ocorrer em diversos grupos musculares, mas em nenhum dos animais deste estudo afetavam os masseteres e a língua. A calcificação não é uma característica observada em eqüinos intoxicados por AIs (Hulland 1993), e não ocorreu nos animais deste estudo.

A intoxicação por Senna occidentalis em eqüinos provoca um quadro principalmente hepatotóxico. Lesões musculares em geral são menos intensas que na intoxicação por Als, sendo observadas apenas na microscopia (Irigoyen et al. 1991). Nos animais deste estudo as lesões eram predominantemente musculares, sendo marcantes e vistas macroscopicamente em vários animais, embora estivessem ausentes em alguns.

Clinicamente a intoxicação por AIs pode ser confundida com cólica, laminite e paralisia periódica hipercalêmica. 
Eqüinos intoxicados por Als apresentam ocasionalmente sinais iniciais de cólica que, no entanto, são geralmente seguidos por distúrbios locomotores, o que diferencia de outras causas de cólica (Van Amstel \& Guthrie 1986). A laminite geralmente ocorre em casos isolados e, muitas vezes, associada a toxinas bacterianas derivadas de distúrbios gastrointestinais ou metrites e retenção de placenta. Ingurgitamento de vasos da lâmina sensitiva do casco pode ser perceptível. A laminite é mais comum em cavalos alimentados com grãos (Radostits et al. 1994).

A paralisia hipercalêmica periódica afeta principalmente equiinos quarto de milha, sendo transmitida geneticamente. Animais afetados normalmente não apresentam sinais clínicos. Quando presentes, os sinais se caracterizam por fasciculações musculares, sudorese, prolapso da terceira pálpebra, inspiração estridente e, ocasionalmente, cólica, fraqueza e cianose. Exposição súbita ao frio, transporte, exercícios físicos vigorosos não costumeiros e eventos estressantes são considerados fatores desencadeantes. A maioria dos ataques são passageiros e as mortes raras (Naylor 1997).

A patogenia das lesões musculares causadas pelos AIs envolve três mecanismos, não autoexcludentes: 1$)$ sobrecarga de cálcio (Wrongemann \& Pena 1976), 2) toxicidade por catecolaminas (Novilla 1992), ou 3) peroxidação das membranas por radicais livres (RLs) (Van Vleet \& Ferrans 1984). Os Als causam influxo celular de sódio e efluxo de potássio para o interstício, seguido pelo influxo de cálcio (Novilla 1992). Para manter a homeostase intracelular, a mitocôndria seqüiestra o excesso de cálcio, resultando em sobrecarga desse íon na mitocôndria, com posterior liberação para o sarcoplasma (Wrongemann \& Pena 1976). O aumento de cálcio no citossol ativa fosfolipases e enzimas proteolíticas que danificam as membranas celulares (Langston et al. 1985) e ATPases que reduzem o estoque de ATP (Wrongemann \& Pena 1976). Alguns Als provocam a liberação de catecolaminas que, através dos produtos de sua oxidação (RLs), provocam peroxidação lipídica das membranas e adicional influxo celular de cálcio (Novilla 1992). O excesso de cálcio no sarcoplasma induz a hipercontração e coagulação das proteínas contráteis, e, em consequiência, necrose (Hulland 1993).

Monensina parece ter efeito cumulativo limitado. $\mathrm{O}$ poder cumulativo de uma substância está relacionado à velocidade de sua absorção comparada à velocidade de excreção. Tendem a se acumular no organismo substâncias absorvidas rapidamente e eliminadas lentamente. Neste experimento, quatro doses diárias de $1 \mathrm{mg} / \mathrm{kg}$ (total de $4 \mathrm{mg} / \mathrm{kg}$ ) reproduziram a doença no eqüino $\mathrm{F}$, mas quarenta doses diárias de 0,5 $\mathrm{mg} / \mathrm{kg}$ (total de $20 \mathrm{mg} / \mathrm{kg}$ ) não a reproduziram no eqüino $\mathrm{G}$. Esses dados são corroborados por estudos sobre velocidade de eliminação da monensina (Davison et al. 1986). Segundo esses estudos, $90-98 \%$ da monensina administrada oralmente a pôneis em doses únicas de $1 \mathrm{mg} / \mathrm{kg}$, são eliminadas em 3 a 4 dias. A eliminação ocorre principalmente pelas fezes, com menores quantidades sendo eliminadas pela urina e bile. Esses dados sugerem que quantidades de $1 \mathrm{mg} / \mathrm{kg} / \mathrm{dia}$ podem atingir a dose letal de $3-4 \mathrm{mg} / \mathrm{kg}$, o que não ocorreria com doses de $0,5 \mathrm{mg} / \mathrm{kg}$.

\section{REFERÊNCIAS}

Amend J.F., Mallon F.M., Wren W.B. \& Ramos A.S. 1980. Equine monensin toxicosis: some experimental clinicopathologic observations. Compendium on Continuing Education 2:173-183.

Armién A.G., Peixoto P.V., Döbereiner J. \& Tokarnia C.H. 1997. Surto de intoxicação por narasina em suínos. Pesq. Vet. Bras. 17:63-68.

Barros C.S.L. 1998. Intoxicação por antibióticos ionóforos, p. 462-466. In: Riet-Correa F., Schild A.L. \& Méndez M.C. (ed.) Doenças de Ruminantes e Equïinos. Editora Universitária, UFPel, Pelotas, RS. 651p.

Beech J. 1997. Chronic exertional rhabdomyolysis. Veterinary Clinics of North America, Equine Practice 13:145-168.

Bezerra P.S., Driemeier D., Loretti A.P., Riet-Correa F., Kamphues J. \& Barros C.S.L. 1999. Monensin poisoning in Brazilian horses. Vet. Human Toxicol. 41:383-385.

Boemo C.M., Tucker J.C., Huntington P.J., Rawlin G.T. \& Drennen P.W. 1991. Monensin toxicity in horses: An outbreak resulting in the deaths of ten horses. Aust. Eq. Vet. 9:103-106.

Chalmers G.A. 1981. Monensin toxicity in broiler chickens. Can. Vet. J. 22:2122.

Davison K.L., Rowe L.D. \& Witzel D.D. $1986 .{ }^{14} \mathrm{C}$ monensin balance in bilefistulated ponies. J. Anim. Sci. 62:173-178.

Doonan G.R., Brown C.M., Mullaney T.P., Brooks D.B., Ulmanis E.G. \& Slanker M.R. 1989. Monensin poisoning in horses - an international incident. Can. Vet. J. 30:165-169.

Gava A., Wouters A.T.B., Wouters F., Nizgoski L. \& Barros C.S.L. 1997. Intoxicação por salinomicina em bovinos. Pesq. Vet. Bras. 17:127-130.

Gerhards H., Fenner A. \& Schoon H.-A. 1986. Monensin-Vergiftung bei Equiden. Dtsch. Tierärztl. Wschr. 93:281-284.

Gregory D.G., Edwards W.C. \& Stair E.L. 1992. A case of monensin poisoning in ostriches. Vet. Human Toxicol. 34:247.

Hanson L.J., Eisenbeis H.G. \& Givens S.V. 1981. Toxic effects of lasalocid in horses. Am. J. Vet. Res. 42:456-461.

Hausmann R. \& Sasse H.H.L. 1991. Monensin-Natrium-Intoxikation der Equiden. Tierärztl. Umschau 46:127-134.

Hulland T.J. 1993. Muscles and tendons, p. 183-266. In: Jubb K.V.F., Kennedy P.C. \& Palmer N. (ed.) Pathology of Domestic Animals. Vol. 1. 3rd ed. Academic Press, San Diego.

Kamphues J., Meyer H., Liebler E.M. \& Johannsen A. 1990. Klinische Störungen bei Pferden nach Aufnahme Ionophore-haltigen Mischfutters. Dtsch. Tierärztl. Wschr. 97:537-539.

Irigoyen L.F., Graça D.L. \& Barros C.S.L. 1991. Intoxicação por Cassia occidentalis (Leg. Caes.) em eqüinos. Pesq. Vet. Bras. 11:35-44.

Langston V.C., Galey F., Lovell R. \& Buck W.B. 1985. Toxicity and therapeutics of monensin: a review. Vet. Med. 80:75-84.

Matsuoka T. 1976. Evaluation of monensin toxicity in the horse. J. Am. Vet. Med. Assoc. 169:1098-1100.

McCracken M., Buclkes E. \& Bowman L. 1998. Monensin intoxication in five horses. Vet. Pathol. 35:420.

Mollenhauer H.H., Rowe L.D., Cysewski S.J., \& Witzel D.A. 1981. Ultrastructural observations in ponies after treatment with monensin. Am. J. Vet. Res. 42:35-39.

Mollenhauer H.H., Rowe L.D. \& Witzel D.A. 1984. Effect of monensin on the morphology of mitochondria in rodent and equine striated muscle. Vet. Human Toxicol. 26:15-19.

Muylle E., Vandenhende C., Oyaert W., Thoonen H. \& Vlaeminck K. 1981. Delayed monensin sodium toxicity in horses. Eq. Vet. J. 13:107-108.

Naylor J.M. 1997. Hyperkalemic periodic paralysis. Veterinary Clinics of North America, Equine Practice 13:129-144.

Nicpon J., Czerw P., Harps O. \& Deegen E. 1997. Salinomycinvergiftung in einem polnischen Pferdebestand [Salinomycin poisoning in a polish stud]. Tierärztl. Praxis 25:438-444. 
Novilla M.N. 1992. The veterinary importance of the toxic syndrome induced by ionophores. Vet. Human Toxicol. 34:66-70.

Nuytten J., Bruynooghe D., Muylle E., Van den Hende C., Vlaeminck K. \& Oyaert W. 1981. Acute en subacute verschijnselen bij monensin intoxicatie bij paarden. Vlaams Diergeneeskd. Tijdschr. 50:242-249.

Ordidge R.M., Schubert F.K. \& Stoker J.W. 1979. Death of horses after accidental feeding of monensin. Vet. Rec. 104: 375.

Radostits O.M., Blood D.C. \& Gay C.C. 1994. Veterinary Medicine. 8th ed. Baillière Tindall, London. 1763p.

Riet-Correa G., Barros C.S.L., Ilha M.R.S. \& Stigger A.L. 1999. Intoxicação por antibiótico ionóforo em ovinos. Anais XIV Jornada Acadêmica de Pesquisa, Universidade Federal de Santa Maria, p. 712.

Rollinson J., Taylor F.G.R. \& Chesney J. 1987. Salinomycin poisoning in horses. Vet. Rec. 121:126-128.

Rooney J.R. \& Robertson J.N. 1996. Equine Pathology. Iowa State University Press, Iowa. 482p.

Salles M.W.S., Barros C.S.L. \& Barros S.S. 1994. Ionophore antibiotic (narasin) poisoning in rabbits. Vet. Human Toxicol. 36:437-444.
Valberg S.J. 1996. Muscular causes of exercise intolerance in horses. Veterinary Clinics of North America, Equine Practice 12:495-515.

Van Amstel S.R. \& Guthrie A.J. 1986. Salinomycin poisoning in horses: case report. Proc. 31st Annual Convention Am. Assoc. Eq. Pract., p. 373-381.

Van de Kerk P.1978. Monensin poisoning in horses. Tijdschr. Diergeneeskd. 103:699-700.

Van Vleet J.F. \& Ferrans V.J. 1984. Ultrastructural alterations in the skeletal muscle of pigs with acute monensin myotoxicosis. Am. J. Pathol. 114:461471.

Whitlock R.H. 1990. Feed additives and contaminants as a cause of equine disease. Veterinary Clinics of North America, Equine Practice 6: 467-478.

Whitlock R.H., White N.A., Rowland G.N. \& Plue R. 1978. Monensin toxicosis in horses: clinical manifestations. Proc. 31st Annual Convention Am. Assoc. Eq. Pract., p. 467-478.

Wilson J.S. 1980. Toxic myopathy in a dog associated with the presence of monensin in dry food. Can. Vet. J. 21:30-31.

Wrongemann K \& Pena S.J. D. 1976. Mitochondrial calcium overload: a general mechanism for necrosis in muscle diseases. Lancet 1: 672-674. 